\title{
POWER MANAGEMENT STRATEGY FOR A PARALLEL HYBRID ELECTRIC TRUCK
}

\author{
Chan-Chiao Lin* Huei Peng* J.W. Grizzle ${ }^{\dagger}$ \\ *Dept. of Mechanical Engineering, University of Michigan, MI 48109-2125, hpeng@umich.edu \\ ${ }^{\dagger}$ Dept. of Electrical Engineering and Computer Science, University of Michigan, MI 48109-2122
}

Keywords: Hybrid Electric Vehicle, Power Management

\begin{abstract}
Hybrid vehicle techniques are widely studied recently because of their potential to significantly improve the fuel economy and drivability of future ground vehicles. Due to the dual-power-source nature of these vehicles, control strategies based on engineering intuition frequently fail to fully explore the potential of these advanced vehicles. In this paper, we will present a procedure for the design of a near-optimum power management strategy. The design procedure starts by defining a cost function, such as minimizing fuel consumption and selected emission species. The Dynamic Programming (DP) techniques are then utilized to find the optimal control actions. Through analysis of the behavior of the DP control actions, sub-optimal rules are extracted, which, unlike DP control signals, are implementable. The performance of the power management control strategy is verified by using the hybrid vehicle model HE-VESIM developed at the Automotive Research Center of the University of Michigan. A trade-off study between fuel economy and emissions was performed. It was found that significant emission reduction can be achieved at the expense of small increase in fuel consumption.
\end{abstract}

\section{Introduction}

Medium and heavy trucks running on diesel engines serve an important role in modern societies. More than $80 \%$ of the freight transported in the US in 1999 was carried by medium and heavy trucks. The increasing reliance on the trucking transportation brings with it certain negative impact. First, the petroleum consumption used in the transportation sector was one of the leading contributors for import oil gap. Furthermore, diesel-engine vehicles are known to be more polluting than gasoline-engine vehicles, in terms of NOx (Nitrogen Oxides) and PM (Particulate Matters) emissions. Recently, hybrid electric vehicle (HEV) technology was proposed as the basis for new vehicle configurations. Owing to their dual on-board power sources and possibility of regenerative braking, HEVs offer unprecedented potential for higher fuel economy while meeting tightened emissions standard, particularly when a parallel configuration is employed. To fully realize the potential of hybrid powertrains, the power management function of these vehicles must be carefully designed. The "power management" function refers to the design of the higher-level, low-bandwidth control algorithm that determines the proper power level to be generated, and its split between the two power sources. In general, the control sampling time for the power management control system is low $(\sim 1 \mathrm{~Hz})$. Its command then becomes the setpoints for the servo-loop control systems, which operate at a much higher frequency $(>20 \mathrm{~Hz})$. The servo-loop control systems can be designed for different goals, such as improved drivability, while ensuring the set-points commanded by the main loop controller are achieved reliably.

Power management strategies for parallel HEVs can be roughly classified into three categories. The first type employs intelligent control techniques such as control rules/fuzzy logic/neural network for estimation and control algorithm development ([1], [2]). The second approach is based on static optimization methods. Commonly, electric power is translated into an equivalent amount of (steady-state) fuel rate in order to calculate the overall fuel cost ([3], [4]). The optimization scheme then figures out proper split between the two energy sources using steady-state efficiency maps. Because of the simple point-wise optimization nature, it is possible to extend such optimization schemes to solve the simultaneous 
Proceedings of the 2002 Mediterranean Control Conference, Lisbon, Portugal, July 2002.

fuel economy and emission optimization problem [5]. The basic idea of the third type of HEV control algorithms consider the dynamic nature of the system when performing the optimization ([6],[7]). Furthermore, the optimization is with respect to a time horizon, rather than for an instance in time. In general, a power split algorithm resulting from dynamic optimization approaches are more accurate under transient conditions, but are more computation-intensive.

In this paper, we apply the Dynamic Programming (DP) technique to solve the optimal power management problem of a hybrid electric truck. The optimal power management solution over a driving cycle is obtained by minimizing a defined cost function. Two cases are solved: a fueleconomy only case, and a fuel/emission case. The comparison of these two cases provides insight into the change needed when the additional objective of emission reduction is included. However, the DP control actions are not implementable due to their preview nature and heavy computational requirement. They are, on the other hand, benchmarks other control strategies can compare against and learn from. We study the behavior of the dynamic programming solution carefully, and extract implementable rules. These rules are used to improve a simple, intuition-based algorithm. It was found that the performance of the intuition (rule) based algorithm improves significantly, and in many cases approaches the DP optimal results.

The paper is organized as follows: In Section 2, the hybrid electric truck model is described, followed by the explanation of the preliminary rule-based control strategy. The dynamic optimization problem and the DP procedure are introduced in Section 3. The optimal results for the fuel consumption and fuel/emissions optimization cases are given in Section 4. Section 5 described the design of improved rule-based strategies. Finally, conclusions are presented in Section 6.

\section{Simulation Model (HE-VESIM)}

2.1 System Configuration

The baseline vehicle studied is the International 4700 series, a 4X2 Class VI truck. The diesel engine was downsized from V8 (7.3L) to V6 (5.5L) and then augmented by a $49 \mathrm{KW} \mathrm{DC}$ electric motor. An 18Amp-hour advanced valveregulated lead-acid (VRLA) battery was chosen as the energy storage system. The hybrid truck was estimated to be $246 \mathrm{~kg}$ heavier than the original design. A schematic of the vehicle is given in Figure 1. The downsized engine is connected to the torque converter (TC), which in turn connects to the transmission (Trns). The transmission and the electric motor are linked to the propeller shaft (PS), differential (D) and two driveshafts (DS). Important parameters of this vehicle are given in Table 1.

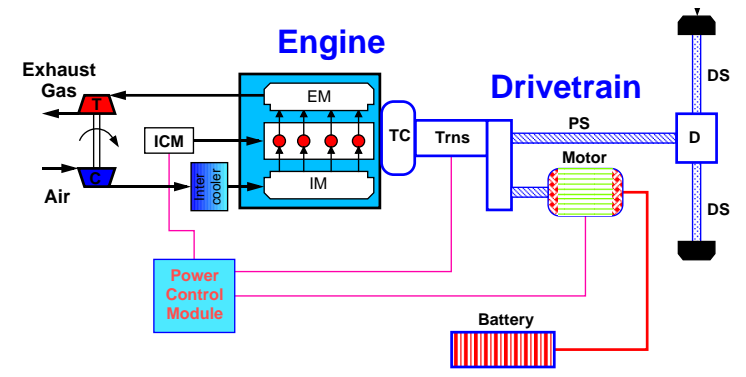

Figure 1: Schematic diagram of the hybrid electric truck

\begin{tabular}{|l|l|}
\hline DI Diesel Engine & V6, 5.475L, $157 \mathrm{HP} / 2400 \mathrm{rpm}$ \\
\hline DC Motor & $49 \mathrm{~kW}$ \\
\hline \multirow{4}{*}{ Lead-acid Battery } & Capacity: $18 \mathrm{Ah}$ \\
\cline { 2 - 2 } & Module number: 25 \\
\cline { 2 - 2 } & Energy density: $34(\mathrm{Wh} / \mathrm{kg})$ \\
\cline { 2 - 2 } & Power density: $350(\mathrm{~W} / \mathrm{kg})$ \\
\hline Automatic Transmission & 4 speed, GR: $3.45 / 2.24 / 1.41 / 1.0$ \\
\hline Vehicle & Curb weight: $7504 \mathrm{~kg}$ \\
\hline
\end{tabular}

Table 1 Basic vehicle parameters

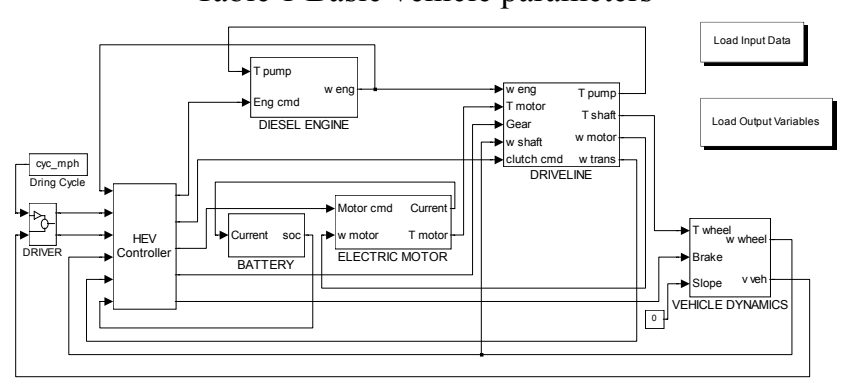

Figure 2: Vehicle model in SIMULINK

The Hybrid Engine-Vehicle SIMulation (HEVESIM) model used in this paper is based on the conventional vehicle model VESIM developed at the University of Michigan [8]. VESIM was validated against measurements for a Class VI truck for both engine operation and vehicle launch/driving performance. The major changes from VESIM include the reduction of the engine 
Proceedings of the 2002 Mediterranean Control Conference, Lisbon, Portugal, July 2002.

size/power and corresponding fuel/emission map, and the integration of the electric components. The HE-VESIM model is implemented in SIMULINK, as presented in Figure 2. Since the model has been presented before ([8], [9]), details are omitted here.

\subsection{Preliminary Rule Based Control Strategy}

Many existing HEV power management algorithms are rule-based, because of the ease in handling switching operating modes. For parallel hybrid vehicles, there are five possible operating modes: motor only, engine only, power-assist (engine plus motor), recharging (engine charges the battery) and regenerative braking. Using motor to start the engine occurs within short period of time and thus is not treated as a regular operating mode. In order to improve fuel economy and/or to reduce emissions, the power management controller has to decide which operating mode to use, and if proper, to determine the optimal split between the two power sources while meeting the driver demand and maintaining battery state of charge. The simple rule-based power management strategy to be presented below was developed on the basis of engineering intuition and simple analysis of component efficiency tables/charts [9, 10], a very popular design approach. The design process starts by interpreting the driver pedal motion as a power request, $P_{\text {req }}$. The operation of the controller is determined by three simple rules: Braking rule, Power Split rule and Recharging rule. If $P_{r e q}$ is negative, The Braking rule is applied to decelerate the vehicle. If $P_{r e q}$ is positive, either Power Split or Recharging rule will be applied, depending on the battery state of charge (SOC). A high-level charge-sustaining strategy tries to maintain the battery SOC within defined lower and upper bounds. A 55-60\% SOC range is chosen for efficient battery operation as well as to prevent battery depletion or damage. It is important to note that these SOC levels are not hard bounds and excursions could occur. Under normal propulsive driving condition, the Power Split rule determines the power flow in the hybrid powertrain. Whenever the SOC drops below the lower limit, the controller will switch to the Recharging rule until the SOC reaches the upper limit, and then Power Split rule will take over. The basic logic of each control rule is described below.

Power Split Control: Based on the engine efficiency map (Figure 3), an "engine on" power line, $P_{e_{-} o n}$, and "motor assist" power line, $P_{m_{-} a}$, are chosen to avoid engine operation in inefficient areas. If $P_{\text {req }}$ is less than $P_{e_{-} o n}$, the electric motor will supply the requested power alone. Beyond $P_{e_{-} \text {on }}$, the engine becomes the sole power source.

Once $P_{\text {req }}$ exceeds $P_{m_{-} a}$, engine power is set at $P_{m_{-} a}$ and the motor is activated to make up the difference $\left(P_{r e q}-P_{m_{-} a}\right)$.

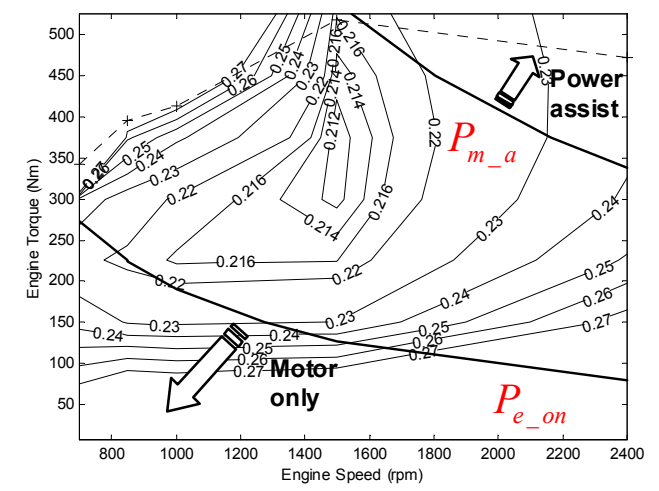

Figure 3: Power Split Control rule

Recharging Control: In addition to power the vehicle, the engine sometimes needs to provide additional power to charge the battery. Commonly a pre-selected recharge power level, $P_{c h}$, is added to the driver's power request which becomes the total requested engine power. The motor power command becomes negative $\left(P_{m}=-P_{c h}\right)$. However, this simple rule is frequently found to be inefficient, and exceptions must be allowed. One example is that when $P_{\text {req }}$ is less than $P_{e_{-} o n}$, the recharging mode might not be activated. If SOC is not excessively low, the motor will still propel the vehicle to prevent inefficient engine operation. The other exception is that when $P_{r e q}$ is greater than $P_{m_{\_} a}$, the motor power will become positive to assist the engine, or stay at zero (when SOC is too low).

Braking Control: When $P_{r e q}$ is negative, regenerative braking is activated. If $P_{\text {req }}$ exceeds the regenerative braking capacity $P_{m \text { min }}$, friction brakes will assist the deceleration $\left(P_{b}=P_{\text {req }}-P_{m_{-} \text {min }}\right)$. 


\subsection{Fuel Economy and Emissions Evaluation}

Unlike light-duty hybrid vehicles, heavy-duty hybrid vehicles do not yet have a standardized test procedure for measuring their emissions and fuel economy performance. A test protocol is under development by SAE and NAVC based on SAE $\mathrm{J} 1711$

[15] when we write this paper. Therefore, we decided to adopt the procedures proposed in [16]. The chassis-based driving schedule for heavy-duty vehicles (UDDSHDV), as opposed to an engineonly dynamometer cycle is adopted. For UDDSHDV, emissions are recorded and reported in the unit of gram per mile $(\mathrm{g} / \mathrm{mi})$. In addition, the battery SOC correction procedure proposed in [16] is used to correct fuel economy and emissions. The hybrid electric truck with the preliminary rule-based controller was tested through simulation over the UDDSHDV cycle. Table 2 compares the results of the HEV with those of the conventional diesel engine truck. It can be seen that the hybrid-electric truck, under the preliminary rule based control algorithm, achieves $27 \%$ better fuel economy compared to the baseline diesel truck. PM reduction is also achieved even though no emission criterion is explicitly included, due to the trickling-down effect of improved fuel economy. NOx level increases because the engine works harder. In fact, this is exactly the main point of this paper: it is hard to include more than one objective in simple rule-based control strategies, which is commonly driven by intuition and trial-and-error. The simple control strategy is not optimal since it is usually component-based as oppose to system-based. Usually we do not even know how much room is left for improvement. This motivates the use of Dynamic Programming as an analysis and design tool.

\begin{tabular}{|c|c|c|c|}
\hline & FE (mi/gal) & NOx (g/mi) & PM (g/mi) \\
\hline Conventional Truck & 10.343 & 5.3466 & 0.5080 \\
\hline $\begin{array}{c}\text { Hybrid Truck } \\
\text { (Preliminary Rule-Base) }\end{array}$ & 13.159 & 5.7395 & 0.4576 \\
\hline
\end{tabular}

Table 2: Performance comparison: conventional vs. HEV

\section{Dynamic Optimization Problem}

Contrary to rule-based algorithms, the dynamic optimization approach relies on a dynamic model to compute the best control strategy. For a given driving cycle, the optimal operating strategy to minimize fuel consumption, or combined fuel consumption/emissions can be obtained. A numerical-based Dynamic Programming (DP) approach is adopted in this paper to solve this finite horizon optimization problem.

\subsection{Problem Formulation}

In the discrete-time format, a model of the hybrid electric vehicle can be expressed as:

$$
x(k+1)=f(x(k), u(k))
$$

where $u(k)$ is the vector of control variables such as fuel injection rate to the engine, desired output torque from the motor, and gear shift command to the transmission. $x(k)$ is the state vector of the system. The sampling time for this main-loop control problem is selected to be one second. The optimization goal is to find the control input, $u(k)$, which minimizes a cost function, which consists of the weighted sum of fuel consumption and emissions for a given driving cycle. The cost function to be minimized has the following form:

$$
J=\sum_{k=0}^{N-1} L(x(k), u(k))=\sum_{k=0}^{N-1} f u e l(k)+\mu \cdot N O x(k)+v \cdot P M(k)
$$

where $N$ is the duration of the driving cycle, and $L$ is the instantaneous cost including fuel use and engine-out NOx and PM emissions. For a fuelonly problem, $\mu=v=0$, and $\mu>0, v>0$ for a simultaneous fuel/emission problem.

During the optimization, it is necessary to impose certain inequality constraints to ensure safe/smooth operation of the engine/battery/motor. The four (or more precisely, eight) constraints we imposed are:

$$
\begin{aligned}
& \omega_{e_{-\min }} \leq \omega_{e}(k) \leq \omega_{e_{-\max }} \\
& T_{e_{-} \text {min }}\left(\omega_{e}(k)\right) \leq T_{e}(k) \leq T_{e_{-\max }}\left(\omega_{e}(k)\right) \\
& T_{m_{-} \text {min }}\left(\omega_{m}(k), S O C(k)\right) \leq T_{m}(k) \leq T_{m_{-} \max }\left(\omega_{m}(k), S O C(k)\right) \\
& S O C_{\min } \leq S O C(k) \leq S O C_{\max }
\end{aligned}
$$

where $\omega_{e}$ is the engine speed, $T_{e}$ is the engine torque, $T_{m}$ is the motor torque and $S O C$ is the battery state of charge. In addition, we also impose two equality constraints for the optimization problem, so that the vehicle always meets the speed and load (torque) demands of the driving cycle at each sampling time. 
Proceedings of the 2002 Mediterranean Control Conference, Lisbon, Portugal, July 2002.

The above problem formulation does not impose any constraint on terminal SOC, the optimization algorithm tends to deplete the battery in order to attain minimal fuel consumption. Hence, a terminal constraint on SOC needs to be imposed:

$$
\begin{aligned}
J & =\sum_{k=0}^{N-1}[L(x(k), u(k))]+G(x(N)) \\
& =\sum_{k=0}^{N-1}[\text { fuel }(k)+\mu \cdot N O x(k)+v \cdot P M(k)]+\alpha\left(S O C(N)-S O C_{f}\right)^{2}
\end{aligned}
$$

where $S O C_{f}$ is the desired SOC at the final time (which is usually equal to the initial SOC), and $\alpha$ is a positive weighting factor.

\subsection{Model Simplification}

The detailed HE-VESIM model (24 states) is not suitable for dynamic optimization because its high computation demand. Due to the selection of the sampling time $(\mathrm{T}=1 \mathrm{sec})$, dynamics that are much faster than $1 \mathrm{~Hz}$ could be ignored. By analyzing the dynamic modes, it was determined that only two state variables need to be kept: the transmission gear number and the battery SOC. The simplifications of the five sub-systems: engine, driveline, transmission, motor/battery and vehicle are described below.

\subsubsection{Engine}

The engine dynamics are ignored and the output torque generated is from a look-up table with two independent variables: engine speed and fuel injected per cylinder/cycle [8]. The feed-gas NOx and PM emissions are functions of engine torque and engine speed and are obtained by scaling the emission maps from the Advisor program [17].

\subsubsection{Driveline}

The driveline components are fast and thus were reduced to static models.

$$
\begin{gathered}
T_{p}=\left(\frac{\omega_{e}}{K\left(\omega_{r}\right)}\right)^{2}, \quad T_{t}=T_{r}\left(\omega_{r}\right) \cdot T_{p} \\
T_{x}=\left(T_{t}-T_{x, l 1}-T_{x, l 2}\left(\omega_{t}, g_{x}\right)\right) \cdot R_{x}\left(g_{x}\right) \cdot \eta_{x}\left(g_{x}\right) \\
T_{d}=\left(T_{x}+R_{c} \cdot T_{m} \cdot \eta_{c}-T_{d, l}\left(\omega_{x}\right)\right) \cdot R_{d} \cdot \eta_{d}
\end{gathered}
$$

where $T_{p}$ and $T_{t}$ are pump and turbine torques, $K$ and $T_{r}$ are the capacity factor and torque ratio of the torque converter, $\omega_{r}=\omega_{t} / \omega_{e}$ is the speed ratio of the torque converter, $T_{x}$ and $T_{d}$ are the output torque of the transmission and differential, respectively. $R_{x}$ and $\eta_{x}$ are gear ratio and efficiency of the transmission, which are functions of the gear number, $g_{x}$

\subsubsection{Transmission}

The automatic transmission is modelled as a ratio device with gear number as the sole state. The control ('shift') to the transmission is constrained to take on the values of $-1,0$, and 1 , representing downshift, sustain and up-shift, respectively. The gear shift dynamics are then described by:

$$
g_{x}(k+1)=g_{x}(k)+\operatorname{shift}(k)
$$

\subsubsection{Motor/Battery}

The electric motor characteristics are based on the efficiency data obtained from the Advisor program [17] as shown in Figure 4. The efficiency of the motor is a function of motor torque and motor speed, $\eta_{m}=f\left(T_{m}, \omega_{m}\right)$. However, due to the battery power and motor torque limit, the final motor torque becomes:

$$
T_{m}=\left\{\begin{array}{l}
\min \left(T_{m, r e q}, T_{m, d i s}\left(\omega_{m}\right), T_{\text {bat dis }}\left(S O C, \omega_{m}\right)\right) T_{m, \text { req }}>0 \\
\max \left(T_{m, \text { req }}, T_{m, \text { chg }}\left(\omega_{m}\right), T_{\text {bat }, \text { h } h g}\left(S O C, \omega_{m}\right)\right) T_{m, \text { req }}<0
\end{array}\right.
$$

where $T_{m, r e q}$ is the requested motor torque, $T_{m, d i s}$ and $T_{m, c h g}$ are the maximum motor torque in the motoring and charging modes, and $T_{b a t, d i s}$ and $T_{\text {bat,chg }}$ are the torque bounds due to battery current limit in the discharging and charging modes.

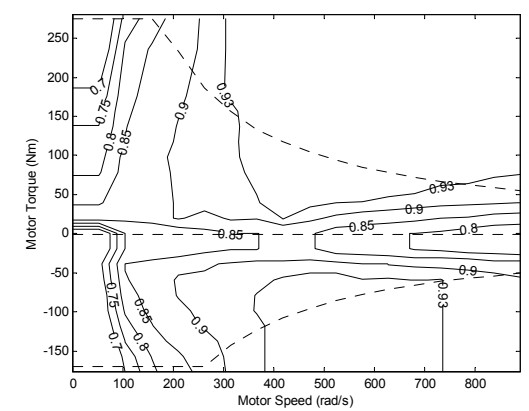

Figure 4: Efficiency map of the DC motor

Of all the sub-systems, the battery is perhaps the least understood. The reason is that the battery performance-voltage, current and efficiency as manifested from a pure electric viewpoint, is the outcome of thermally-dependent electrochemical 
Proceedings of the 2002 Mediterranean Control Conference, Lisbon, Portugal, July 2002.

processes that are not quite complicated. Various models have been developed in the literature. But if we ignore thermal-temperature and transient effects (due to internal capacitance), the battery model reduces to a static equivalent circuit shown in Figure 5. The only state variable left in the battery is the state of charge (SOC):

$$
\operatorname{SOC}(k+1)=\operatorname{SOC}(k)-\frac{V_{o c}-\sqrt{V_{o c}^{2}-4\left(R_{\mathrm{int}}+R_{t}\right) \cdot T_{m} \cdot \omega_{m} \cdot \eta_{m}}}{2\left(R_{\mathrm{int}}+R_{t}\right) \cdot Q_{b}}
$$

where the internal resistance $R_{\text {int }}$ and the open circuit voltage $V_{o c}$ are functions of the battery SOC, $Q_{b}$ is the maximum battery charge and $R_{t}$ is the terminal resistance. The battery plays an important role in the overall performance of $\mathrm{HEV}$ because of its nonlinear, non-symmetric and relatively low efficiency characteristics. Figure 6 shows the charging and discharging efficiency of the battery. It can be seen that discharging efficiency decreases at low SOC and charging efficiency decreases at high SOC region. Overall, the battery operates more efficiently at low power levels in both charging and discharging.

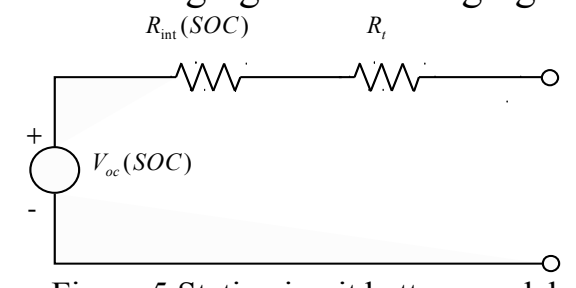

Figure 5 Static-circuit battery model
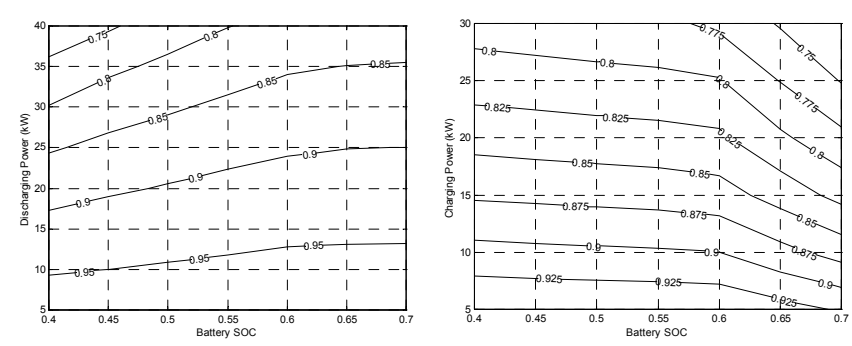

Figure 6: Efficiency maps of the lead acid battery: discharging (left) and charging (right)

\subsubsection{Vehicle}

The vehicle is modelled as a pointmass:

$v_{v}(k+1)=v_{v}(k)+\frac{1}{M_{r}}\left(\frac{T_{w h}(k)}{r_{d}}-\frac{B_{w h} v_{v}(k)}{r_{d}^{2}}-\frac{v_{v}(k)}{\left|v_{v}(k)\right|}\left(F_{r}+F_{a}\left(v_{v}(k)\right)\right)\right)(11)$

where $v_{v}$ is the vehicle speed, $T_{w h}$ is the net wheel torque from driveline and hydraulic brake, $r_{d}$ is the dynamic tire radius, $B_{w h}$ is the viscous damping, $F_{r}$ and $F_{a}$ are the rolling resistance force and the aerodynamic drag force, $M_{r}=M_{v}+J_{r} / r_{d}^{2}$ is the effective mass of the vehicle and $J_{r}$ is the equivalent moment of inertia of the rotating components in the vehicle.

\subsection{Dynamic Programming Method}

Based on Bellman's principle of optimality, the DP solution for the cost function shown in Eq.(4) is [11]:

Step $N-1$ :

$$
J^{*}{ }_{N-1}(x(N-1))=\min _{u(N-1)}[L(x(N-1), u(N-1))+G(x(N))](
$$

Step $k$, for $0 \leq k<N-1$

$$
J^{*}{ }^{*}(x(k))=\min _{u(k)}\left[L(x(k), u(k))+J^{*}{ }_{k+1}(x(k+1))\right]
$$

The recursive equation is solved backwards to find the optimal control policy, subject to the inequality constraints shown in Eq. (3) and the equality constraints imposed by the driving cycle. A standard way to solve the above stated DP problem numerically is to use quantization and interpolation ([11], [12]). The state and control values are first quantized into finite grids. At each step of the optimization search, the function $J_{k}(x(k))$ is evaluated only at the grid points of the state variables. If the next state, $x(k+1)$, does not fall exactly on to a quantized value, then the values of $J^{*}{ }_{k+1}(x(k+1))$ in Eq.(13) as well as $G(x(N))$ in (12) are determined through interpolation.

Despite the use of a simplified model, and a quantized search space, the long time horizon makes the above algorithm computationally expensive. In this research, we adopted two "tricks" to acceleration the optimization search. First, from the velocity profile of the driving cycle, the required wheel torque $T_{w h, r e q}$ is determined by inversely solving Eq.(11). The wheel speed $\omega_{w h, r e q}$ can be computed by feeding the required wheel torque to the vehicle model in order to include the wheel dynamics and slip effect. Combining this procedure with the defined state/input grid, the vehicle model can be replaced by a finite set of operating points parameterized by $T_{w h, r e q}$ and $\omega_{w h, r e q}$. The second trick adopted is to construct pre-computed look-up tables for the new states and instantaneous cost as a function of 
Proceedings of the 2002 Mediterranean Control Conference, Lisbon, Portugal, July 2002.

quantized states, control inputs, and operating points. Once these tables are built, they can be used to update Eq.(13) efficiently by the vector operations in MATLAB [12].

\section{Dynamic Programming Results}

The DP procedure described above produces an optimal, time-varying, state-feedback control policy, i.e., $u^{*}(x(k), k)$. It should be noted that DP creates a family of optimal paths for all possible initial conditions. Once the initial SOC is specified, the optimal policy will find a way to achieve the minimal weighted cost of fuel consumption and emissions while bringing the final SOC close to the desired terminal value $\left(S O C_{f}\right)$. The optimal control policy was applied to the HE-VESIM model for final evaluation. In the following, two cases are presented: fuel economy only, and simultaneous fuel/emission optimization.

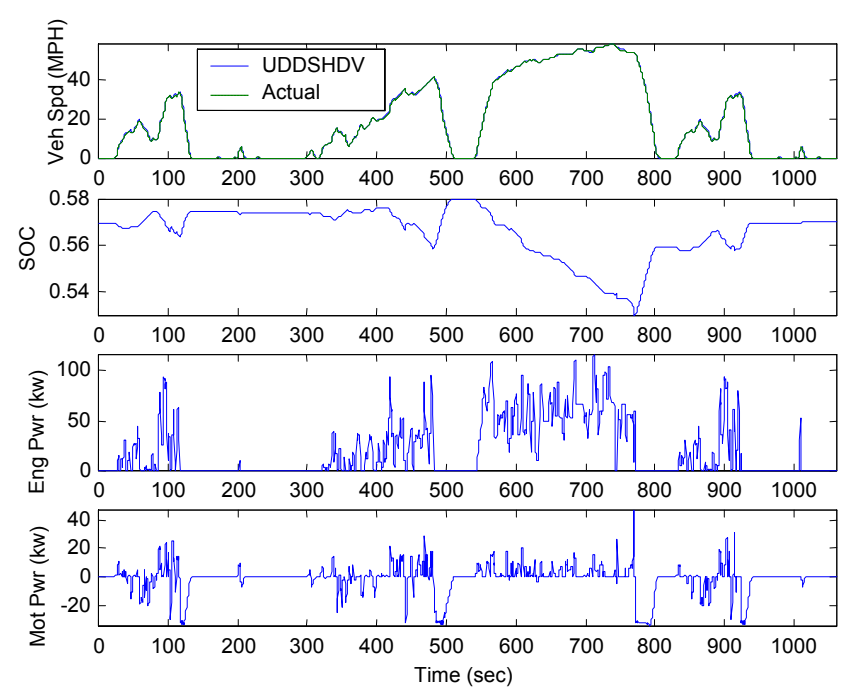

Figure 7 Simulation results for the fuel-economy-only case

\subsection{Fuel Economy optimization results}

The weightings in Eq.(4) are chosen to be $\mu=0, v=0, \alpha=5 \cdot 10^{6}$ for this case. The UDDSHDV driving cycle is again used. The initial and terminal SOC were both selected to be 0.57. Simulation results of the vehicle under the DP policy are shown in Figure 7. There is a small difference $(<2 \mathrm{mph})$ between the desired vehicle speed (UDDSHDV) and the achieved vehicle speed, caused by model mismatch and the long sampling time $(1 \mathrm{sec})$. The engine power and motor power trajectories represent the optimal operation between two power movers for achieving the best fuel economy. Additional $6 \%$ fuel economy improvement was achieved by the DP algorithm (Table 3) as compared with values shown in Table 2.

\begin{tabular}{|c|c|c|c|c|}
\hline & FE (mi/gal) & Fuel (g/mi) & NOx (g/mi) & PM (g/mi) \\
\hline$\mu=0, v=0$ & 13.705 & 234.71 & 5.627 & 0.446 \\
\hline \multicolumn{5}{|c}{ Table 3: Summary of DP results }
\end{tabular}

\subsection{Fuel Economy and Emissions Optimization}

To study the trade-off between fuel economy and emissions, the weighting factors are varied:

$$
\begin{aligned}
& \mu \in\{0,5,10,20,40\} \\
& v \in\{0,100,200,400,600,800\}
\end{aligned}
$$

Selected optimization results are shown in Figures 8 and 9. The case of $\mu=v=0$ corresponds to the optimal fuel economy scenario. Figure 8 shows the trade-off in NOx emissions and fuel economy. Increasing $\mu$ leads to significant NOx reduction while causing a small fuel economy increase. Increasing $v$ results in reduced PM (Figure 9) but higher NOx emissions and lower fuel economy (Figure 8). The trade-off between NOx and PM can be seen from Figure 9 where larger $v$ tends to decrease PM emission but increase NOx emission. More importantly, significant reduction in NOx and PM emissions can be achieved at the price of a small increase in fuel consumption.

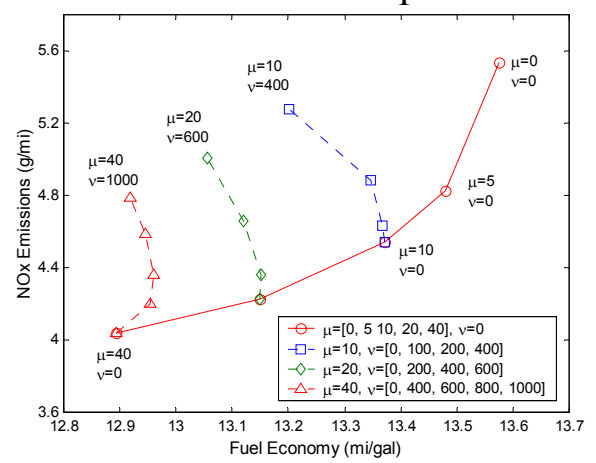

Figure 8 Fuel economy versus engine-out NOx emissions The case $\mu=40, v=800$ seems to achieve a good trade-off--NOx and PM are reduced by $17.3 \%$ and $10.3 \%$ respectively at a $3.67 \%$ penalty on fuel economy. Simulation results of this case are shown in Figure 10. The SOC fluctuates in a wider range compared to the fuel-only case (Figure 6). It can be seen that for the fuel-only case, almost all the negative motor power are from 
Proceedings of the 2002 Mediterranean Control Conference, Lisbon, Portugal, July 2002.

regenerative braking. In other words, the engine seldom recharges the battery. Therefore, all electrical energy consumed comes from regenerative braking. This implies that it is not efficient to use engine power to charge the battery. This is due to the fact that the fuel efficiency map of this diesel engine is flat in medium to high power regions.

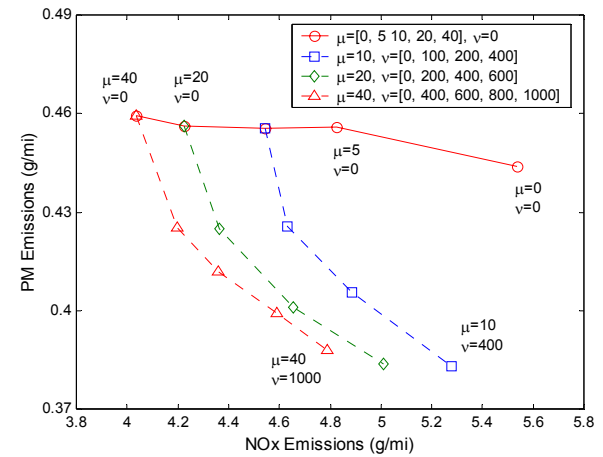

Figure 9 Engine-out PM emissions versus NOx emissions

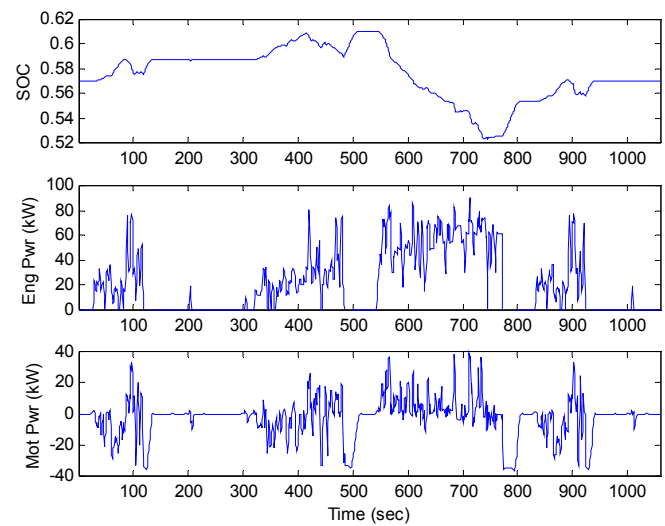

Figure 10 Simulation results $(\mu=40, v=800)$

\section{Development of Improved Rule-Based Controls}

The DP control policy is not implementable in real driving conditions because it requires knowledge of future speed and load profile. Nonetheless, analyzing its behaviour provides useful insight into possible improvement.

\subsection{Gear Shift Control}

Gear shifting strategy is crucial to the fuel economy of hybrid electric vehicles. The original optimal gear trajectory has frequent shifting which is undesirable. Hence, a drivability constraint is added to avoid frequent gear shifting:

$$
\begin{aligned}
J & =\sum_{k=0}^{N-1}\left(f u e l(k)+40 \cdot \operatorname{NOx}(k)+800 \cdot P M(k)+\beta \cdot\left|g_{x}(k+1)-g_{x}(k)\right|\right) \\
& +5 \cdot 10^{6} \cdot\left(\operatorname{SOC}(N)-S O C_{f}\right)^{2}
\end{aligned}
$$

where $\beta$ is a positive weighting factor. Figure 11 shows the optimal gear position trajectories from DP for different $\beta$ values. It can be seen that larger $\beta$ results in less-frequent gear shifting. The value of $\beta=1.5$ is chosen. From the DP results, the gear operational points are expressed on the engine power demand vs. transmission speed plot (Figure 12). It can be seen that the gear positions are separated into four regions and the boundary between adjacent regions represent optimal gear shifting thresholds. After adding a hysteresis function to the shifting thresholds, a new gear shift map is obtained. It should be mentioned that the optimal gear shift map can also be constructed through static optimization ([10], [14]). Given an engine power and wheel speed, the best gear position for minimum weighted cost of fuel and emissions can be chosen based on the combined steady-state engine fuel consumption and emissions map. It is found that the steadystate gear map from this method nearly coincides with Figure 12.

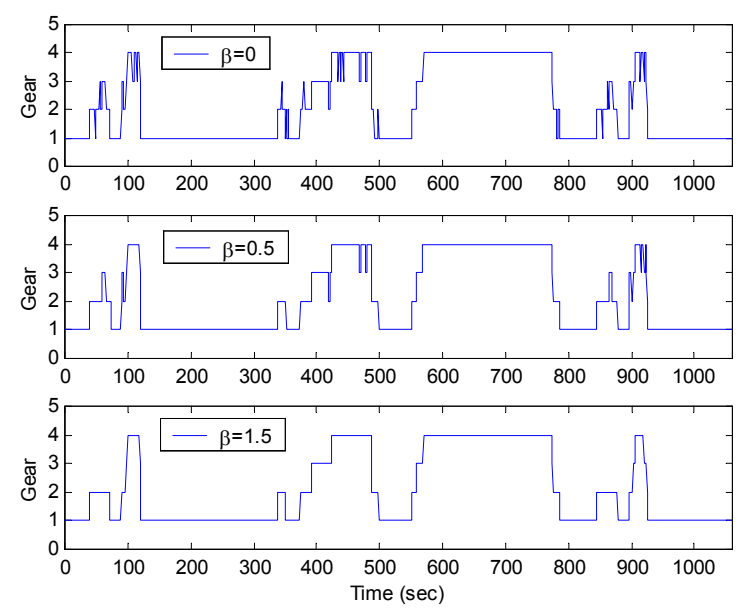

Figure 11 Optimal gear position trajectory

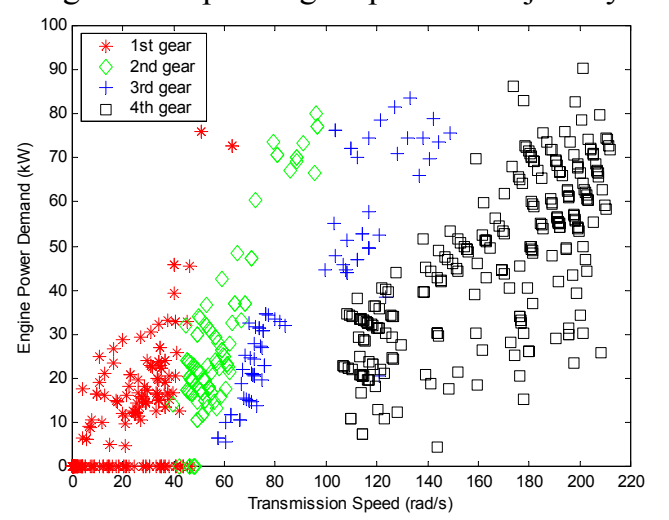

Figure 12 Gear operating points of DP optimization 


\subsection{Power Split Control}

In this section, we study how Power Split Control of the preliminary rule-based strategy can be improved. A power-split-ratio $P S R=\frac{P_{\text {eng }}}{P_{\text {req }}}$ is defined to quantify the positive power flows in the powertrain, where $P_{\text {eng }}$ is the engine power and $P_{\text {req }}$ is the power request from the driver. Four positive-power operating modes are defined: motor-only $(P S R=0)$, engine-only $(P S R=1)$, power-assist $(0<P S R<1)$, and recharging mode $(P S R>1)$. The optimal (DP) behavior uses the motor-only mode in low power-demand region when the vehicle launches. When the wheel speed is above $6 \mathrm{rad} / \mathrm{s}$, a simple rule is found by plotting the optimal PSR versus the power request over the transmission input speed, which is equivalent to torque demand at the torque converter output shaft (see Figure 13). The figure shows the optimal policy uses the recharging mode $(P S R>1)$ in the low torque region, the engine-only mode in the middle torque region, and the power-assist mode in the high torque region. This can be explained by examining a weighted Brake Specific Fuel and Emissions Consumption (BSFEC) of the engine.

$$
B S F E C=\frac{W_{f}+\mu \cdot W_{N O x}+v \cdot W_{P M}}{P_{e n g}}
$$

The contour of engine BSFEC map is shown in the Figure 14. It can be seen that the best BSFEC region occurs at low torque level. In order to move the engine operating points towards a better BSFEC region, the engine is used to recharge the battery at low load, and the motor is used to assist the engine at high load. In order to extract an implementable rule, a least-square curve fit is used to approximate the optimal PSR, shown as the solid line in Figure 13.

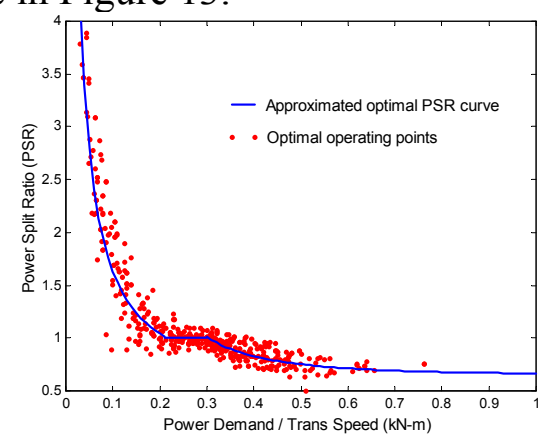

Figure 13 DP power split behavior (UDDSHDV cycle)

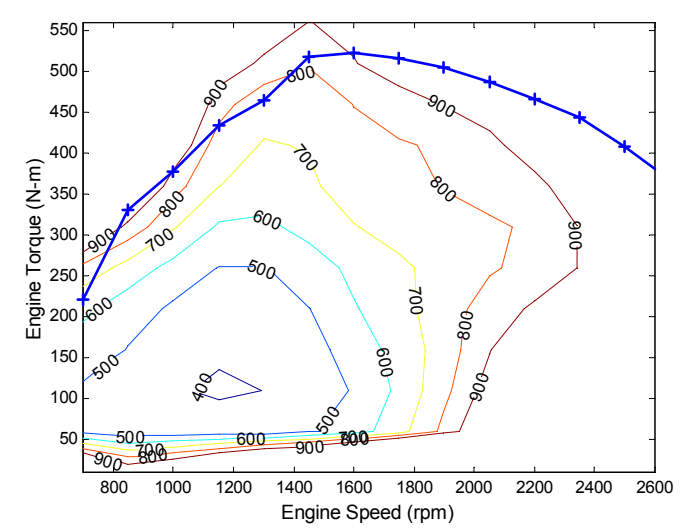

Figure 14 BSFEC map in $\mathrm{g} / \mathrm{kWhr}$

\subsection{Charge-Sustaining Strategy}

The power split control scheme described above does not maintain the battery SOC within desired operating range. The DP procedure is repeated again except this time the regenerative braking function is turned off. The curve-fitted optimal PSR result is compared with the one with regenerative braking in Figure 15. More aggressive spending of battery energy can be used when SOC is high and more conservative rules can be used when SOC is low. These adaptive PSR rules can be learned from DP results by specifying different initial SOC points.

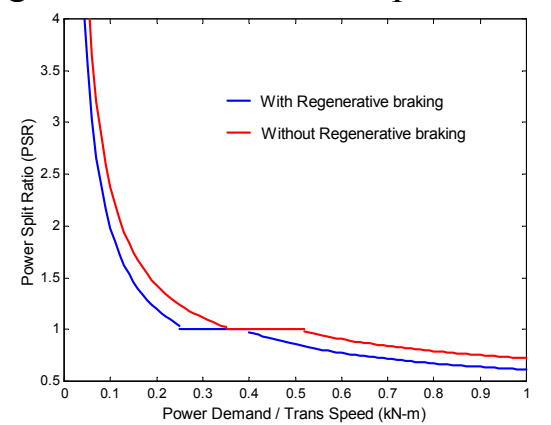

Figure 15 Optimal PSR rules comparison

\subsection{Performance Evaluation}

After incorporating all the changes outlined in the previous sections, the improved rule-based controller is evaluated using several different driving cycles. In addition to the original cycle (UDDSHDV), the new rule-based controller is put through three other driving cycles (suburban, interstate, and city) to test its robustness. The results are shown in Tables 4-7. It can be seen that depending on the nature of the driving cycles, the new rule-based control system may not improve all three categories of performance, and in certain cases did worse. However, if the 
Proceedings of the 2002 Mediterranean Control Conference, Lisbon, Portugal, July 2002.

combined fuel/emission performance is considered (the "performance measure"), the new rule-based controller is always significantly better than the original, intuition driven rule-based control law.

\begin{tabular}{|c|c|c|l|c|}
\hline & FE (mi/gal) & NOx (g/mi & PM (g/mi) & $\begin{array}{l}\text { Performance } \\
\text { Measure }\end{array}$ \\
\hline Baseline Rule-Based & 13.159 & 5.7395 & 0.4576 & 840.63 \\
\hline New Rule-Based & 12.8738 & 4.8355 & 0.4292 & 787.0965 \\
\hline DP (FE \& Emis) & 13.237 & 4.6422 & 0.3992 & 739.56 \\
\hline
\end{tabular}

Table 4: Results over the UDDSHDV cycle

Performance Measure: fuel $+40 \cdot N O x+800 \cdot P M(\mathrm{~g} / \mathrm{mi})$

\begin{tabular}{|c|c|c|c|c|}
\hline & FE (mi/gal) & $\begin{array}{c}\text { NOx } \\
(\mathrm{g} / \mathrm{mi})\end{array}$ & $\begin{array}{c}\text { PM } \\
(\mathrm{g} / \mathrm{mi})\end{array}$ & $\begin{array}{c}\text { Performance } \\
\text { Measure }\end{array}$ \\
\hline $\begin{array}{c}\text { Baseline Rule- } \\
\text { Based }\end{array}$ & 15.3103 & 4.4291 & 0.3547 & 671.225 \\
\hline New Rule-Based & 14.5839 & 2.9273 & 0.2959 & 574.6322 \\
\hline DP (FE \& Emis) & 15.4108 & 2.7785 & 0.2585 & 526.666 \\
\hline
\end{tabular}

Table 5 Results over the WVUSUB cycle

\begin{tabular}{|c|c|c|c|c|}
\hline & FE (mi/gal) & NOx (g/mi) & PM (g/mi) & $\begin{array}{c}\text { Performance } \\
\text { Measure }\end{array}$ \\
\hline Baseline Rule-Based & 12.8433 & 7.2850 & 0.5086 & 948.8256 \\
\hline New Rule-Based & 12.7198 & 6.2733 & 0.4878 & 894.106 \\
\hline DP (FE \& Emis) & 12.9658 & 6.1675 & 0.4411 & 847.6675 \\
\hline
\end{tabular}

Table 6 Results over the WVUINTER cycle

\begin{tabular}{|c|c|c|c|c|}
\hline & FE (mi/gal) & NOx (g/mi) & PM (g/mi) & $\begin{array}{c}\text { Performance } \\
\text { Measure }\end{array}$ \\
\hline Baseline Rule-Based & 16.1791 & 3.8698 & 0.3320 & 621.2218 \\
\hline New Rule-Based & 15.3649 & 2.4091 & 0.2187 & 480.7421 \\
\hline DP (FE \& Emis) & 16.6257 & 2.0367 & 0.1608 & 403.578 \\
\hline
\end{tabular}

Table 7 Results over the WVUCITY cycle

\section{Conclusions}

Designing the power management strategy for HEV by learning from the Dynamic Programming (DP) results has the clear advantage of being nearoptimal, accommodates multiple objectives, and systematic. Depending on the overall objective, one can easily develop power management laws that emphasize fuel economy, and/or emissions. By analyzing the DP results, improved rule-based control strategy can be developed. The learned behavior was found to be robust, rather than cyclespecific. This is evident by the fact that the learned behavior based on one cycle works extremely well for several never-seen driving cycles, moving the rule-based control law closer to the theoretical optimal (DP results) by $50-70 \%$.

\section{Acknowledgments}

This research is supported by the U.S. Army TARDEC under the contract DAAE07-98-C-RL008. The work of J.W. Grizzle was supported in part by NSF contract IIS-9988695 .

\section{References}

[1]Baumann, B. M. et al. "Mechatronic Design and Control of Hybrid Electric Vehicles," IEEE/ASME Transactions on Mechatronics, v5 n 1 2000. p 58-72, 2000

[2]Farrall, S. D. and Jones, R. P., "Energy Management in an Automotive Electric/Heat Engine Hybrid Powertrain Using Fuzzy Decision Making," Proceedings of the 1993 International Symposium on Intelligent Control, Chicago, IL, 1993

[3]Kim, C., NamGoong, E., and Lee, S., "Fuel Economy Optimization for Parallel Hybrid Vehicles with CVT," SAE Paper No. 1999-01-1148.

[4]Paganelli, G. et al. "A General Formulation for the Instantaneous Control of the Power Split in Charge-Sustaining Hybrid Electric Vehicles." Proceedings of 5th Int'l Symposium on Advanced Vehicle Control, Ann Arbor, MI, 2000.

[5]Johnson, V.H., Wipke, K.B. and Rausen, D.J., "HEV Control Strategy for Real-Time Optimization of Fuel Economy and Emissions," Proceedings of the Future Car Congress, April 2000, SAE Paper No. 2000-01-1543.

[6]Brahma, A., et al. "Dynamic Optimization of Mechanical Electrical Power Flow in Parallel Hybrid Electric Vehicles" Proc. of 5th Int'l Sypm. Adv. Veh. Ctrl, Ann Arbor, MI, 2000.

[7]Zoelch, U., and Scroeder, D., "Dynamic Optimization Method for Design and Rating of the Components of a Hybrid Vehicle," International Journal of Vehicle Design, v19, n1, p1-13, 1998

[8]Assanis, D.N. et al. "Validation and Use of SIMULINK Integrated, High Fidelity, Engine-In-Vehicle Simulation of the International Class VI Truck," SAE Paper No. 2000-01-0288

[9]Lin, C.C., Filipi, Z.S., Wang, Y., Louca, L.S., Peng, H., Assanis, D.N., and Stein, J.L., "Integrated, Feed-Forward Hybrid Electric Vehicle Simulation in SIMULINK and its Use for Power Management Studies", SAE Paper No. 2001-01-1334

[10]Bowles, P. D., "Modeling and Energy Management for a Parallel Hybrid Electric Vehicle (PHEV) with Continuously Variable Transmission (CVT)," MS thesis, University of Michigan, Ann Arbor, MI, 1999

[11]Bertsekas, D.P., Dynamic Programming and Optimal Control, Athena Scientific, 1995

[12]Jun-Mo Kang et al. “Approximate Dynamic Programming Solutions for Lean Burn Engine Aftertreatment," Proc. of the IEEE Conf. on Dec. and Ctrl, Phoenix, AZ, Dec. 7-10, 1999

[13]Lee, H.D. et al. "Advanced Gear Shifting and Clutching Strategy for Parallel Hybrid Vehicle with Automated Manual Transmission," IEEE Ind. Appl. Conference, v3, 1998

[14]Soltic, P., and Guzzella, L., "Optimum SI Engine Based Powertrain Systems for Lightweight Passenger Cars," SAE Paper No. 2000-01-0827, 2000

[15] Society of Automotive Engineers, Hybrid-Electric Vehicle test Procedure Task Force, "SAE J1711, Recommended Practice for Measuring Exhaust Emissions and Fuel Economy of Hybrid-Electric Vehicles," 1998

[16] Mckain, D. L. et al. "Characterization of Emissions from Hybrid-Electric and Conventional Transit Buses," SAE Paper 2000-01-2011, 2000

[17] National Renewable Energy Laboratory, "ADVISOR 3.2 Documentation," http://www.ctts.nrel.gov/analysis/, 2001. 